\title{
Expression of TPM1 $\kappa$, a Novel Sarcomeric Isoform of the TPM1 Gene, in Mouse Heart and Skeletal Muscle
}

\author{
Syamalima Dube, Lauren Panebianco, Amr A. Matoq, Henry N. Chionuma, \\ Christopher R. Denz, Bernard J. Poiesz, and Dipak K. Dube
}

Department of Medicine, SUNY Upstate Medical University, 750 East Adams Street, Syracuse, NY 13210, USA

Correspondence should be addressed to Dipak K. Dube; dubed@upstate.edu

Received 25 October 2013; Revised 5 February 2014; Accepted 6 February 2014; Published 24 April 2014

Academic Editor: Jamboor Vishwanatha

\begin{abstract}
Copyright (C) 2014 Syamalima Dube et al. This is an open access article distributed under the Creative Commons Attribution License, which permits unrestricted use, distribution, and reproduction in any medium, provided the original work is properly cited.

We have investigated the expression of TPM1 $\alpha$ and TPM1 $\kappa$ in mouse striated muscles. TPM1 $\alpha$ and TMP1 $\kappa$ were amplified from the cDNA of mouse heart by using conventional RT-PCR. We have cloned the PCR amplified DNA and determined the nucleotide sequences. Deduced amino acid sequences show that there are three amino acid changes in mouse exon $2 \mathrm{a}$ when compared with the human TPM1 $\kappa$. However, the deduced amino acid sequences of human TPM1 $\alpha$ and mouse TPM1 $\alpha$ are identical. Conventional RT-PCR data as well as qRT-PCR data, calculating both absolute copy number and relative expression, revealed that the expression of TPM1 $\kappa$ is significantly lower compared to TPM1 $\alpha$ in both mouse heart and skeletal muscle. It was also found that the expression level of TPM1 $\kappa$ transcripts in mouse heart is higher than it is in skeletal muscle. To the best of our knowledge, this is the first report of the expression of TPM1 $\kappa$ in mammalian skeletal muscle.
\end{abstract}

\section{Introduction}

Tropomyosins (TMs) are a family of highly conserved actin binding proteins which are expressed in all eukaryotes from yeast to humans. TMs play a critical role in the control of $\mathrm{Ca}^{+2}$-regulated thin filament function in striated muscle contraction. Except for zebrafish, in vertebrates, there are four known tropomyosin (TPM) genes (designated as TPM1, TPM2, TPM3, and TPM4) [1-6]. In zebrafish, six tropomyosin genes have been reported [7]. Each of the TPM genes generates a multitude of tissue and developmental specific isoforms via alternate splicing. TPM1 is known to produce at least ten alternatively spliced transcript variants [8].

In mammals, the predominant striated muscle isoform is TPM1 $\alpha$ containing exons $1 \mathrm{a}, 2 \mathrm{~b}, 3,4,5,6 \mathrm{~b}, 7,8$, and $9 \mathrm{a} / \mathrm{b}$, which encode 284 amino acid residues. Our laboratory first reported another striated muscle isoform known as TPM1 $\kappa$ in axolotl heart [9] that contains exons 1a, 2a (instead of exon 2b), 3, 4, 5, 6b, 7, 8, and 9a/b. We also reported the expression of TPM1 $\kappa$ in axolotl skeletal muscle [8], human heart [10], and embryonic chicken heart [11]. In humans, TPM1 $\kappa$ is expressed in both fetal and adult hearts, whereas in chicken both TPM1 $\alpha$ and TPM1 $\kappa$ are expressed only in embryonic heart [11]. Interestingly, transgenic mice that overexpressed human $\mathrm{TPM} 1 \kappa$, in a cardiac-specific manner, were found to develop a dilated cardiomyopathy-like syndrome [12]. However, it was not clear whether TPM1 $\kappa$ is expressed in normal mouse hearts. Two possible reasons for observing some abnormalities in TG mouse hearts ectopically overexpressing human TPMl $\kappa$ were that too much TPMl $\kappa$ is toxic to the mouse cardiomyocyte or that human TPM1 $\kappa$ is variant from its mouse homologue and, for that reason alone, is toxic. Hence, we decided to explore TPM1 $\kappa$ expression in mouse hearts by RT-PCR and sequencing.

In this study, we report the expression of TPMl $\kappa$ in mouse heart and skeletal muscles. To the best of our knowledge, this is the first report that TPM1 $\kappa$ is expressed in mammalian skeletal muscle. Two different methods were used to quantify the expression of TPMl $\alpha$ and TPM1 $\kappa$. We determined the absolute copy number as well as relative expression compared to a reference gene (18S rRNA) in mouse heart and skeletal muscle. Unlike in humans [12], the expression level of TPM1 $\kappa$ transcripts in mouse heart is much lower compared to 
TABLE 1: Primer pairs and probes used for amplification and detection of TPMl $\alpha$ and/or TPM1 $\kappa$.

\begin{tabular}{|c|c|c|}
\hline Name & Name as in Figure 1 & Sequences \\
\hline Mus TPM $1 \alpha / \kappa-1(+)$ & P1 & $5^{\prime}$-ATGGACGCCATCAAGAAGAA-3' \\
\hline Mus TPM1 $\alpha / \kappa-2(-)$ & $\mathrm{P} 2$ & $5^{\prime}$-TTATATGGAAGTTATATCGTT-3' \\
\hline Mus TPM1 $\alpha-1(+)$ & P3 & $5^{\prime}$-CTCAAGGGCACTGAAGATGAA-3' \\
\hline Mus TPM1 $\kappa-1(+)$ & $\mathrm{P} 4$ & $5^{\prime}$-CTCGAGGAGATCGCAGCG-3' \\
\hline Mus TPM1 $\alpha$ probe & & $5^{\prime}$-CTGGAGCTGGCGGAGAAAAAGGCC-3' \\
\hline Mus TPM1 $\kappa$ probe & P5 & $5^{\prime}$-GGCGGAGGACAGCCTCCTGGCTGC-3' \\
\hline Mus TPM1 UTR (-) & & $5^{\prime}$-GAGTCTTGGGAGGAGTGAAG-3' \\
\hline
\end{tabular}

TPM1 $\alpha$. Further, there are three amino acid changes in mouse relative to human TPM1 $\kappa$.

\section{Materials and Methods}

Mouse whole heart RNA was procured from Stratagen and ZyAgen; skeletal muscle RNA from BioChain and ZyAgen. Also, we received mouse whole heart and skeletal muscle RNA as a generous gift from Dr. David Wieczroek, University of Cincinnati, Cincinnati, $\mathrm{OH}$. Each of these four sources of RNA came from a single adult mouse.

The quality of RNA was determined by capillary electrophoresis using 2100 Bioanalyzer, Agilent Technologies. The RIN (RNA integrity number) of the RNA samples ranged from 7.2 to 9, indicating that the RNAs we used were of good quality.

2.1. Preparation of cDNA for Conventional RT-PCR. $0.5 \mu \mathrm{g}$ of RNA in a total volume of $40 \mu \mathrm{L}$ was used for preparing first strand cDNA synthesis with the SuperScript ${ }^{\mathrm{R}}$ II (Life Technologies, Brand Island, NY) and oligodT primer following the manufacturer's specification. For each PCR amplification, $3 \mu \mathrm{L}$ of cDNA was used $[13,14]$. Nucleotide sequences for the primer pairs for amplification of various mouse genes are given in Table 1 .

2.2. Conventional RT-PCR, Cloning, and Sequencing of

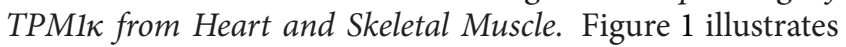
the pattern of alternative splicing of the two sarcomeric TPM1 isoforms. TPMl $\alpha$ contains exons $1 \mathrm{a}, 2 \mathrm{~b}, 3,4,5,6 \mathrm{~b}, 7,8$, and $9 \mathrm{a} / \mathrm{b}$, whereas TPM1 $\kappa$ has all the same exons except it contains exon $2 \mathrm{a}$ instead of exon $2 \mathrm{~b}$. The figure also depicts the strategy for amplification of the two TPM1 isoforms. Primer pair $\mathrm{P} 1(+) / \mathrm{P} 2(-)$ was used to amplify both TPM1 $\alpha$ and TPM1 $\kappa$. Amplification by the primer pairs P3(+)/P2(-) and $\mathrm{P} 4(+) / \mathrm{P} 2(-)$ will yield $\mathrm{TPM} 1 \alpha$ and $\mathrm{TPM} 1 \kappa$, respectively. Subsequently, an isoform-specific [32P]-labeled probe was used for southern hybridization of TPM1 $\alpha$ or TPM1 $\kappa$. The sequences of various primer pairs as well as probes are shown in Table 1 . We amplified TPM1 $\alpha$ and TPM1 $\kappa$ by RTPCR with cDNA from mouse heart muscle using the mus $\mathrm{TPM} 1 \alpha / \kappa$ primer pair. The amplified DNA was run on a $1.5 \%$ agarose gel and the DNA band of $\sim 850 \mathrm{bp}$ was extracted using MinElute Gel Extraction kit of QIAGEN. The eluted DNA was ligated to T/A cloning vector (Invitrogen) following the manufacturer's specification as described earlier [10]. The ligation mix was used for transformation of competent one shot E. coli cells (Invitrogen) using the protocol supplied by Invitrogen. Appropriate hybridization positive colonies were picked up from the plates after filter hybridization with [32P]labeled probe. Exon 2 a specific probe was used for TPM1 $\kappa$ and exon $2 b$ specific probe was used for TPM1 $\alpha$. Colonies were grown overnight with LB medium containing appropriate concentration of antibiotic and plasmid DNA was isolated using QIAprep Spin Miniprep kit (QIAGEN). Isolated plasmid DNA was sequenced at the Cornell University DNA sequencing facility (Figure 5).

2.3. $c D N A$ for $q R T-P C R$. In order to exclude all other isoforms of the TPM1 gene, cDNA was made with a negative primer from exon 9a $\left(5^{\prime}\right.$-CGCTCTCAACGATATGACTT$\left.3^{\prime}\right)$, which is common to both TPM $1 \alpha$ and TPM $1 \kappa$ and is absent from other isoforms (Figure 1). $0.5 \mu \mathrm{g}$ of RNA in a total volume of $40 \mu \mathrm{L}$ was used for preparing the first strand cDNA synthesis with the SuperScript ${ }^{\mathrm{R}}$ II (Life Technologies, Brand Island, NY) following the manufacturer's specification.

2.4. Real Time qRT-PCR. Real-time quantitative RT-PCR (qRT-PCR) analysis of cDNA template was performed using the LightCycler 480 real-time PCR system. Reactions were carried out in a 384-well plate using the LightCycler 480 SYBR Green I Master kit (Roche). Briefly, each well contained a total volume of $10 \mu \mathrm{L}$, of which $2 \mu \mathrm{L}$ was cDNA template and $8 \mu \mathrm{L}$ was SYBR Green mix ( $5 \mu \mathrm{L}$ 1x SYBR Green Master mix, $2.8 \mu \mathrm{L}$ of PCR grade water, and $0.2 \mu \mathrm{L}$ of $10 \mu \mathrm{M}$ primer pair). Expression of TPM1 isoforms was determined using primer pairs for TPM1 $\alpha$ and TPM1 $\kappa,(+) 5^{\prime}$-TGGAAGATGAGCTGGTGTCAC- $3^{\prime} /(-) 5^{\prime}$-TCAATGACTTTCATGCCTCT$3^{\prime}$, and $(+) 5^{\prime}$-CTCGAGGAGGACATCGCAGCG-3' $3^{\prime} /(-) 5^{\prime}-$ TCAATGACTTTCATGCCTCT- $3^{\prime}$, respectively. $18 \mathrm{~S}$ rRNA was assessed as an internal control with primer pair $(+) 5^{\prime}$ GTGGAGCGATTTGTCTGGTT- $3^{\prime} /(-) 5^{\prime}$-CGCTGAGCCAGTCAGTGTAG-3'. Amplification in the absence of cDNA template was also evaluated to ensure lack of signal due to primer dimerization and extension or carryover. All samples and controls were performed in triplicate. End point melt curve analysis confirmed the presence of a single amplicon in each reaction well. A crossing threshold (CT) value was obtained, corresponding to the fractional number of amplification cycles where the PCR curve reaches a program-defined threshold amount of fluorescence. It is to be noted that qRTPCR was performed separately with cDNAs prepared from 


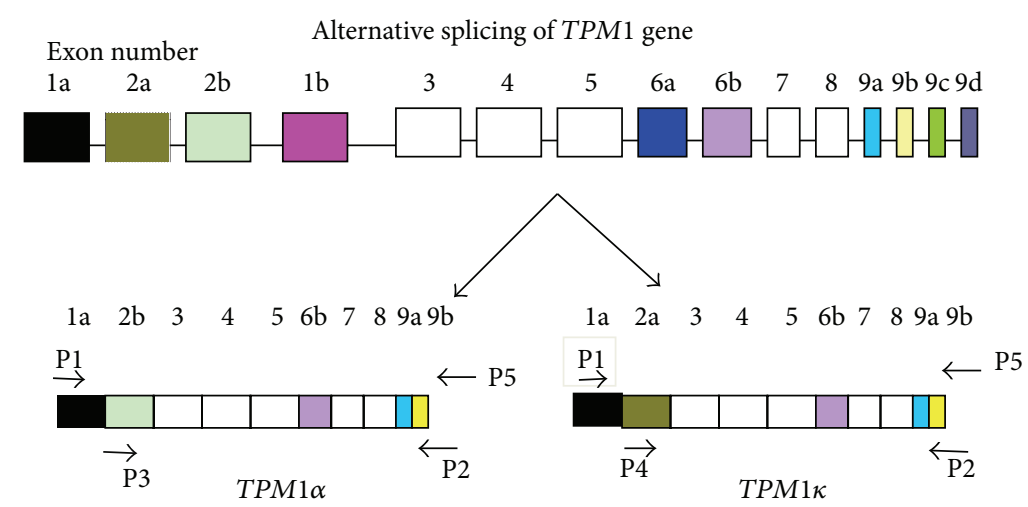

(a)

Exon composition of various high molecular weight TM isoforms

\begin{tabular}{|c|c|c|c|c|}
\hline $\begin{array}{l}\text { TM isoforms } \\
\text { referred to } \\
\text { in this paper }\end{array}$ & $\begin{array}{l}\text { Encoding genes } \\
\text { (old nomenclature) }\end{array}$ & $\begin{array}{l}\text { Isoforms of TM currently } \\
\text { known as }\end{array}$ & Exon composition & $\begin{array}{l}\text { Nomenclature } \\
\text { used in previous } \\
\text { publications }\end{array}$ \\
\hline$T P M 1 \alpha$ & TPM1 $(\alpha-\mathrm{TM})$ & Striated muscle & la, $2 \mathrm{~b}, 3,4,5.6 \mathrm{~b}, 7,8,9 \mathrm{a} / \mathrm{b}$ & ATmC-1/a-Tm-1 \\
\hline$T P M 1 \beta$ & TPM1 $(\alpha-\mathrm{TM})$ & Smooth muscle & $1 \mathrm{a}, 2 \mathrm{a}, 3,4,5,6 \mathrm{~b}, 7,8,9 \mathrm{~d}$ & Sm a-Tm \\
\hline$T P M 1 \gamma$ & TPM1 $(\alpha-\mathrm{TM})$ & TM2- fibroblast & $1 \mathrm{a}, 2 \mathrm{~b}, 3,4,5,6 \mathrm{~b}, 7,8,9 \mathrm{~d}$ & \\
\hline$T P M 1 \delta$ & TPM1 $(\alpha-\mathrm{TM})$ & TM3- fibroblast & $1 \mathrm{a}, 2 \mathrm{~b}, 3,4,5,6 \mathrm{a}, 7,8,9 \mathrm{~d}$ & \\
\hline TPM1к & TPM1 $(\alpha-\mathrm{TM})$ & Striated muscle & $1 \mathrm{a}, 2 \mathrm{a}, 3,4,5,6 \mathrm{~b}, 7,8,9 \mathrm{a} / \mathrm{b}$ & $\mathrm{ATmC}-2 / \mathrm{a}-\mathrm{Tm}-2$ \\
\hline TPM $2 \alpha$ & TPM2 ( $\beta$-TM $)$ & Striated muscle & $1 \mathrm{a}, 2 \mathrm{~b}, 3,4,5,6 \mathrm{~b}, 7,8,9 \mathrm{a} / \mathrm{b}$ & \\
\hline TPM $3 \alpha$ & TPM3 (hTMnm) & Skeletal muscle & $1 \mathrm{a}, 2 \mathrm{~b}, 3,4,5,6 \mathrm{~b}, 7,8,9 \mathrm{a} / \mathrm{b}$ & \\
\hline$T P M 4 \alpha$ & TPM4 (TM4) & StrTM4 & $1 \mathrm{a}, 2 \mathrm{~b}, 3,4.5,6 \mathrm{~b}, 7,8,9 \mathrm{a} / \mathrm{b}$ & ATmC-3/Str.TM-4 \\
\hline
\end{tabular}

(b)

FIGURE 1: Alternate splicing of the TPM1 gene and exon composition of various TM isoforms. (a) Splicing pattern of the TPM1 gene and the strategy for amplification of TPM $1 \alpha$ and TPM1 $\kappa$. Arrows indicate the primer pairs used for amplification of TPM $1 \alpha$ and TPM1 $\kappa$ by RTPCR and subsequent southern hybridization. The nucleotide sequences of various primer pairs and probes are listed in Table 1. (b) Exon composition of various TPM isoforms.

RNA procured from three different sources as mentioned above.

Data were analyzed using both relative and absolute quantification methods. Relative quantification of qRT-PCR data was performed using the delta CT (sample CT minus $18 \mathrm{~S}$ rRNA CT) and delta delta CT (sample delta CT minus comparator delta CT) methods $[15,16]$. A comparative value was calculated using the formula $X^{- \text {delta delta CT }}$, where " $X$ " equals the efficiency of TPM $\alpha$ or TPM1 $\kappa$ primer pairs. This is similar to the $2^{\text {-delta delta CT }}$ method but corrects for the assumption that the reaction is occurring with $100 \%$ efficiency. Efficiencies $(E)$ were determined using dilution series of TPM1 $\alpha$ and TPM1 $\kappa$ plasmid clones with respective isoform-specific primers pairs. The LightCycler 480 software plotted the CT at each concentration against the logarithm of the fold dilution of the clone, generating a linear regression curve that calculated efficiency based on the formula $E=$ $10^{[-1 / \text { slope }]}$. Efficiencies were $79.05 \%$ and $88.42 \%$ for TPM $1 \alpha$ and $\mathrm{TPM} 1 \kappa$, respectively. In instances where TPM $\alpha$ and
TPM1 $\kappa$ were compared within the same cDNA sample, their efficiencies were averaged to equal $83.73 \%$. Efficiency of $18 \mathrm{~S}$ rRNA was determined by serial dilution of mouse cDNAs generated with exon 9a primer.

For determination of absolute copy number, optical density was taken of mouse TPM1 $\kappa$ and TPM1 $\alpha$ TA clones separately using a spectrophotometer. The copy number per volume of clone in solution was determined using the equation number of copies $=($ ng of plasmid DNA $\times 6.02$ $\left.\times 10^{23}\right) /\left(\right.$ bp length of plasmid $\left.\times 1 \times 10^{9} \times 650\right)$, which was simplified by Andrew Staroscik at the URI Genomics and Sequencing Center. A dilution series of each clone was done for $1 \times 10^{1}-1 \times 10^{4}$ copies of template, which was used to create a standard curve after amplification $[8,14]$. For better accuracy, each sample in dilution series was run in triplicate.

2.5. Statistical Analyses. The means, standard deviations, and comparative analyses of each data set for statistical significance were done using paired Student's $t$-test. 


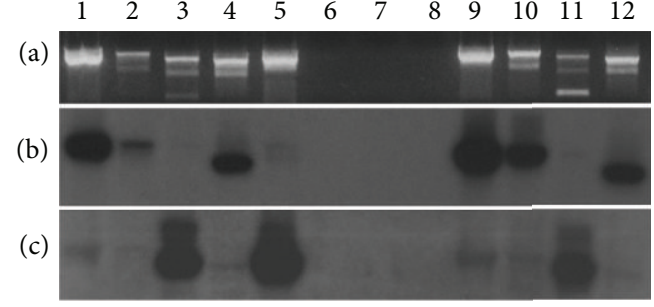

FIGURE 2: Expression analysis of TPM1 $\alpha$ and TPM1 $\kappa$ RNA in mouse heart and skeletal muscle by RT-PCR. Plate (a) illustrates the ethidium bromide stain. Plate (b) illustrates hybridization with TPM1 $\alpha$ probe. Plate (c) illustrates hybridization with TPM1 $\kappa$ probe. Lane 1: heart cDNA amplified with Mus TPM1 $\alpha / \kappa-1(+) /$ Mus UTR $(-)$. Lane 2: heart cDNA amplified with Mus TPM1 $\alpha / \kappa-1(+) /$ Mus TPM1 $\alpha / \kappa-2(-)$. Lane 3: heart cDNA amplified with Mus TPM1 $\kappa-$ $1(+) /$ Mus TPM1 $\alpha / \kappa-2(-)$. Lane 4 : heart cDNA amplified with TPM1 $\alpha(+) /$ Mus TPM1 $\alpha / \kappa-2(-)$. Lane 5: heart cNDA amplified with Mus TPMl $\kappa-1(+) /$ Mus UTR (-). Lane 6: primer control for Mus TPM1 $\alpha / \kappa-1(+) /$ Mus UTR (-). Lane 7: primer control for Mus TPM $1 \alpha / \kappa-1(+) /$ Mus TPM $1 \alpha / \kappa-2(-)$. Lane 8: primer control for Mus TPM1 $\kappa-1(+) /$ Mus TPM1 $\alpha / \kappa-2(-)$. Lane 9: skeletal cDNA amplified with Mus TPM1 $\alpha / \kappa-1(+) /$ Mus UTR (-). Lane 10: skeletal cDNA amplified with Mus TPM1 $\alpha / \kappa-1(+) /$ Mus TPM $1 \alpha / \kappa-2(-)$. Lane 11: skeletal cDNA amplified with Mus TPM1 $\kappa-1(+) /$ Mus TPM $1 \alpha / \kappa-2$ $(-)$. Lane 12: skeletal cDNA amplified with Mus TPM1 $\alpha-1(+) /$ Mus $\mathrm{TPM} 1 \alpha / \kappa-2(-)$.

\section{Results}

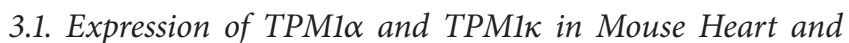
Skeletal Muscle. For expression analysis, we first employed conventional RT-PCR with cDNA made from total RNA from mouse heart and skeletal muscle. TPM1 $\kappa$ RNA was expressed in both mouse heart and skeletal muscle. To the best of our knowledge, this is the first report of the expression of TPM1 $\kappa$ in mammalian skeletal muscle, albeit at a significantly lower level compared to TPM1 $\alpha$.

Figure 2 depicts the results of conventional RT-PCR analysis of TPM1 $\alpha$ and TPM1 $\kappa$ with cDNAs synthesized from mouse heart and skeletal muscle total RNA (provided by Dr. David Wieczroek), using a variety of generic as well as isoform specific primer pairs. Panel (a) in Figure 2 represents the ethidium stained PCR products run on a $1.5 \%$ agarose gel. Lanes 1 and 2 show the PCR products amplified from heart cDNA with two different primer pairs, which amplify both TPM1 $\alpha$ and TPM1 $\kappa$. Southern hybridization with TPM1 $\alpha$ specific probe demonstrated stronger signals in both lanes (lanes 1 and 2, panel (b)) compared to hybridization with TPM1 $\kappa$-specific probe (lanes 1 and 2, panel (c)). The weaker signals in lanes 1 and 2 in panel (c) compared to panel (b) indicate much lower expression level of TPM1 $\kappa$ compared to TPM1 $\alpha$. Lanes 3 and 4 in panel (a) represent the ethidium staining of the PCR products when amplified with TPM1 $\kappa$ or TPM1 $\alpha$-specific primer pairs, respectively. Southern hybridization with TPM $\alpha$-specific probe shows a strong hybridization signal (lane 4, panel (b)) with the TPM $\alpha$ specific primer pair. A strong hybridization signal was also detected with TPM1 $\kappa$-specific probe (lane 3, panel (c)) with the TPM1 $\kappa$-specific primer pair. The results indicate that both

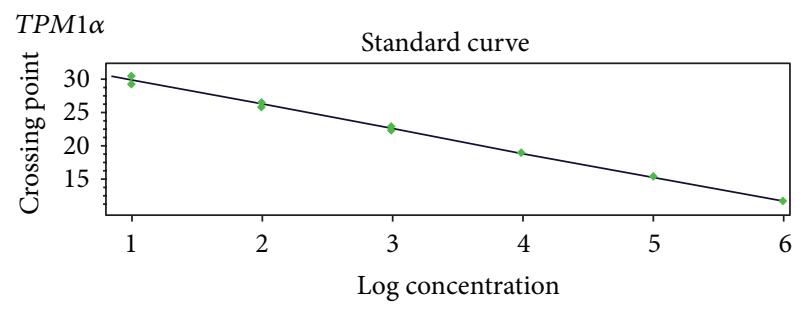

Error: 0.0141; efficiency: 1.873; slope: -3.670 ; $Y$ intercept: 33.53

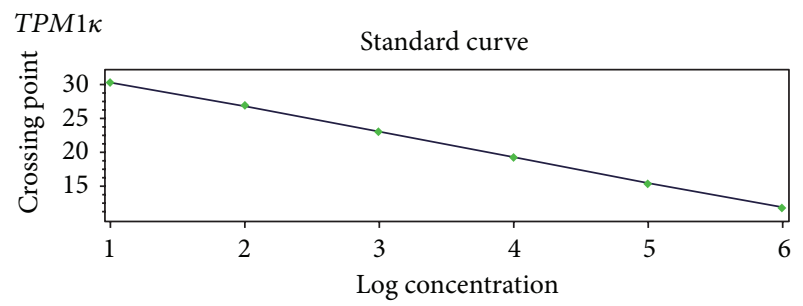

Error: 0.0593; efficiency: 1.856; slope: -3.722 ; $Y$ intercept: 34.00

FIgURE 3: Quantitative RT-PCR of TPM1 $\alpha$ and TPM $1 \kappa$. Standard curve was derived from amplification of mouse TPM1 $\alpha$ and TPM1 $\kappa$ plasmid clones after serial dilution. Copy number of TPM1 $\alpha$ and TPM1 $\kappa$ was calculated per microgram of total RNA from mouse heart and skeletal muscle (Table 2).

TPM1 $\alpha$ and TPM1 $\kappa$ RNA are expressed in mouse heart. This was further confirmed by amplification with another TPM $1 \kappa$ specific primer pair and subsequent southern hybridization with TPM1 $\kappa$-specific probe (lane 5, panels (a), (b), and (c)). All primer controls were negative (lanes 6, 7, and 8). Lanes 9 and 10 represent the PCR amplicons of mouse skeletal muscle cDNA with two sets of TPM $\alpha / \kappa$ primer pairs. Ethidium bromide stained gels indicate a strong expression of TPM1 in skeletal muscle (panel (a)). Hybridization signal with TPM1 $\alpha$-specific probe (panel (b)) versus the TPM1 $\kappa$-specific probe (panel (c)) indicates a much stronger expression of the former. However, amplification of skeletal muscle cDNA with TPM1 $\kappa$-specific primer pair (lane 11 in panel (a)) and subsequent hybridization with TPM1 $\kappa$-specific probe (lane 11 in panel (c)) validate the expression of TPM1 $\kappa$ in mouse skeletal muscle. Similarly, the expression of TPM1 $\alpha$ in skeletal muscle was authenticated by the PCR amplification with TPM1 $\alpha$-specific primer pair (lane 12, panel (a)) and by subsequent hybridization with TPM $\alpha$-specific probe (lane 12, panel (b)). A much lower expression level of TPM1 $\kappa$ compared to TPM $1 \alpha$ in mouse heart and skeletal muscle was further substantiated by qRT-PCR as described below. Similar conventional RT-PCR results were obtained from the RNA procured from Stratagen and Biochain (data not shown).

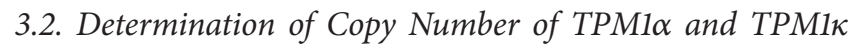
in Mouse Heart and Skeletal Muscles by qRT-PCR. Relative quantification of TPM $1 \alpha$ and TPM1 $\kappa$ in mouse heart and skeletal muscles was performed on all sources of RNA. The standard curves for TPM1 $\alpha$ and TPM1 $\kappa$ are shown in Figure 3. Our qRT-PCR data for copy number determination indicate that the expression of TPM1 $\kappa$ is much lower in both heart and 
TABLE 2: Copy number of TPM1 $\alpha$ and TPM1 $\kappa$ in mouse heart and skeletal muscle.

\begin{tabular}{lccccccccc}
\hline \multirow{2}{*}{ Isoform } & \multicolumn{4}{c}{ Absolute copy number/ $\mu$ g of total RNA $\times 10^{5}$} \\
& Expt 1 & Expt 2 & Expt 3 & Ave & Expt 1 & Expt 2 & Expt 3 & Ave \\
\hline TPM1 $\alpha$ & $7.3 \pm 0.06$ & $5.5 \pm 0.04$ & $7.34 \pm 0.03$ & $6.71 \pm 1.05$ & $19.6 \pm 0.4$ & $23.4 \pm 1.6$ & $15.8 \pm 1.4$ & $19.6 \pm 3.8$ \\
TPM1 $\alpha$ & $0.013 \pm 0.001$ & $0.040 \pm 0.004$ & $0.02 \pm 0.00$ & $0.024 \pm 0.010$ & $0.004 \pm 0.000$ & $0.011 \pm 0.002$ & $0.001 \pm 0.001$ & $0.0055 \pm 0.004$ \\
TPM1 $\alpha:$ TPM1 $\kappa$ & 561 & 137 & 367 & 355 & 4900 & 2127 & 9294 & 5440 \\
\hline
\end{tabular}

(i) TPM1 $\alpha$ heart: TPM1 $\alpha$ skeletal muscle, $P<0.001$.

(ii) TPM1 $\kappa$ heart: TPM1 $\kappa$ skeletal muscle, $P<0.001$.

(iii) TPM1 $\alpha$ heart : TPM1 $\kappa$ heart, $P<0.001$.

(iv) TPM1 $\alpha$ skeletal muscle: TPM1 $\kappa$ skeletal muscle, $P<0.001$.

TABLE 3: Fold changes of TPM1 $\alpha$ and TPM1 $\kappa$ in heart and skeletal muscle (by copy number).

\begin{tabular}{lccccccc}
\hline & \multicolumn{3}{c}{ Ratio of TPM1 $\alpha$ in skeletal muscle : heart } & \multicolumn{4}{c}{ Ratio of TPM1 $\kappa$ in heart : skeletal muscle } \\
Expt 1 & Expt 2 & Expt 3 & Mean \pm SD & Expt 1 & Expt 2 & Expt 3 & Mean \pm SD \\
\hline 2.68 & 4.25 & 2.15 & $3.0 \pm 1.09$ & 3.25 & 3.63 & 10.0 & $5.62 \pm 3.79$ \\
\hline
\end{tabular}

skeletal muscle compared to TPM1 $\alpha$ (Table 2). In relation to cardiac muscle, the data indicate that the level of expression of TPM1 $\alpha$ transcripts is significantly higher and the level of $\mathrm{TPM} 1 \kappa$ is significantly lower in skeletal muscle (Tables 2 and 3).

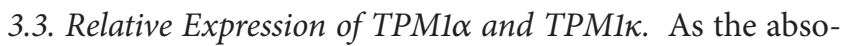
lute copy number determination revealed that the expression level of TPM1 $\kappa$ is significantly lower in heart and skeletal muscle, we analyzed our results by $2^{\wedge}-(\mathrm{ddCT})$ method using $18 \mathrm{~S}$ rRNA as the reference gene to determine the relative expression of TPM1 $\alpha$ and TPM1 $\kappa$. Melt curve data presented in Figure 4(a) show different unique melting temperatures for TPM1 $\alpha$ (left) and TPM1 $\kappa$ (right). Also, agarose gel electrophoresis of the PCR amplified DNA for TPM $\alpha$ or TPM1 $\kappa$ shows a single band for both of them (Figure 4(b)). Hence, each primer pair recognizes only TPM $\alpha$ or TPM $1 \kappa$ amplified DNA.

The data in Table 4 validate our previous findings that expression of TPM $\alpha$ is significantly lower than TPM $\alpha$ in mouse heart and skeletal muscle. The fold changes of TPM1 $\alpha / \mathrm{TPM} 1 \kappa$ are significantly higher in skeletal muscle suggesting the higher expression level of TPM1 $\kappa$ in heart as concluded from the absolute copy number data (Table 2 ). We analyzed our qRT-PCR data by $2^{\wedge}-(\mathrm{ddCT})$. The results support our conclusion that TPM1 $\alpha$ expression is much higher in mouse skeletal versus heart muscle; and the level of expression of TPM1 $\kappa$ is higher in mouse heart versus skeletal muscle (Table 5).

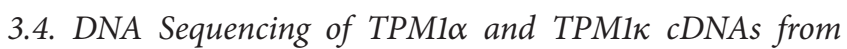
Mouse Heart and Skeletal Muscle. cDNA sequencing confirmed the expression of both TPM1 $\alpha$ and TPM1 $\kappa$ expression in mouse heart and skeletal muscle. Nucleotide and the deduced amino acid sequences of mouse TPM1 $\alpha$ were identical with the published sequence (accession number NM_001164248.1) (data not shown). Also, the nucleotide sequences of mouse TPM1 $\alpha$ and TPM1 $\kappa$ as depicted in Figure 5 are identical except for exon 2. As predicted,
TPM1 $\alpha$ contains exon 2 b, but TPM1 $\kappa$ has exon $2 \mathrm{a}$. The deduced amino acid sequences of mouse and human exon $2 \mathrm{~b}$ of the TPM1 gene are identical. The deduced amino acid sequences of exon $2 \mathrm{a}$ of mouse TPM $1 \kappa$ and the published exon 2 a sequences in mouse TPM1 $\beta$ (smooth muscle TPM1 isoform) (NM_001164249.1) are also identical. However, some differences were noted when comparing the deduced amino acid sequences of mouse exon $2 \mathrm{a}$ with human TPM1 $2 \mathrm{a}$ (Figure 6). Amino acid residues ${ }^{72} \mathrm{E}$ and ${ }^{74} \mathrm{~A}$ in humans are replaced by ${ }^{72} \mathrm{D}$ and ${ }^{74} \mathrm{~T}$ in mouse $\mathrm{TPM} 1 \kappa$, respectively.

However, the most notable difference is at amino acid residue 52. In humans, it is valine, whereas in mouse it is alanine. This amino acid residue is in the middle of the 15mer peptide (KEKLLRVSEDERDRV) that was used as the antigen for developing antibody against human TPM1 $\kappa$ [12]. This alteration may cause a lower affinity of this antibody towards mouse TPM $1 \kappa$ protein. In order to evaluate the expression of TPM $\kappa$ protein, in this study, we carried out western blot analysis with isolated myofibrils from mouse heart and skeletal muscle using the human TPM1 $\kappa$-specific antibodies. The antibodies against human protein showed several nonspecific bands with mouse heart and skeletal muscle extracts. The western results are not convincing as to whether TPM1 $\kappa$ protein is expressed in mouse striated muscle.

\section{Discussion}

We first detected and sequenced TPM1 $\kappa$ from the heart of the Mexican axolotl [9]. Both the RNA and protein of this sarcomeric TM isoform is expressed in both heart and skeletal muscle in axolotl [8]. Subsequently, we reported the expression of TPM1 $\kappa$ transcripts and protein in human heart but not in skeletal muscle [10]. Also, TPM1 $\kappa$ along with TPM1 $\alpha$ is expressed in embryonic chicken heart but not in skeletal muscle [11]. However, the transcripts of neither TM isoform could be detected in adult chicken heart and skeletal muscle [11]. Our present study clearly demonstrates that TPM $1 \kappa$ RNA is expressed in adult mouse 


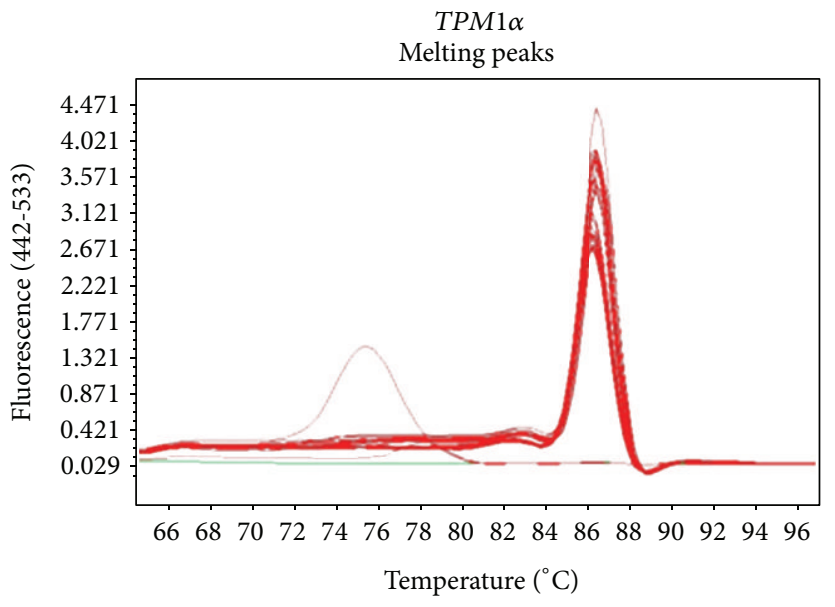

(a)
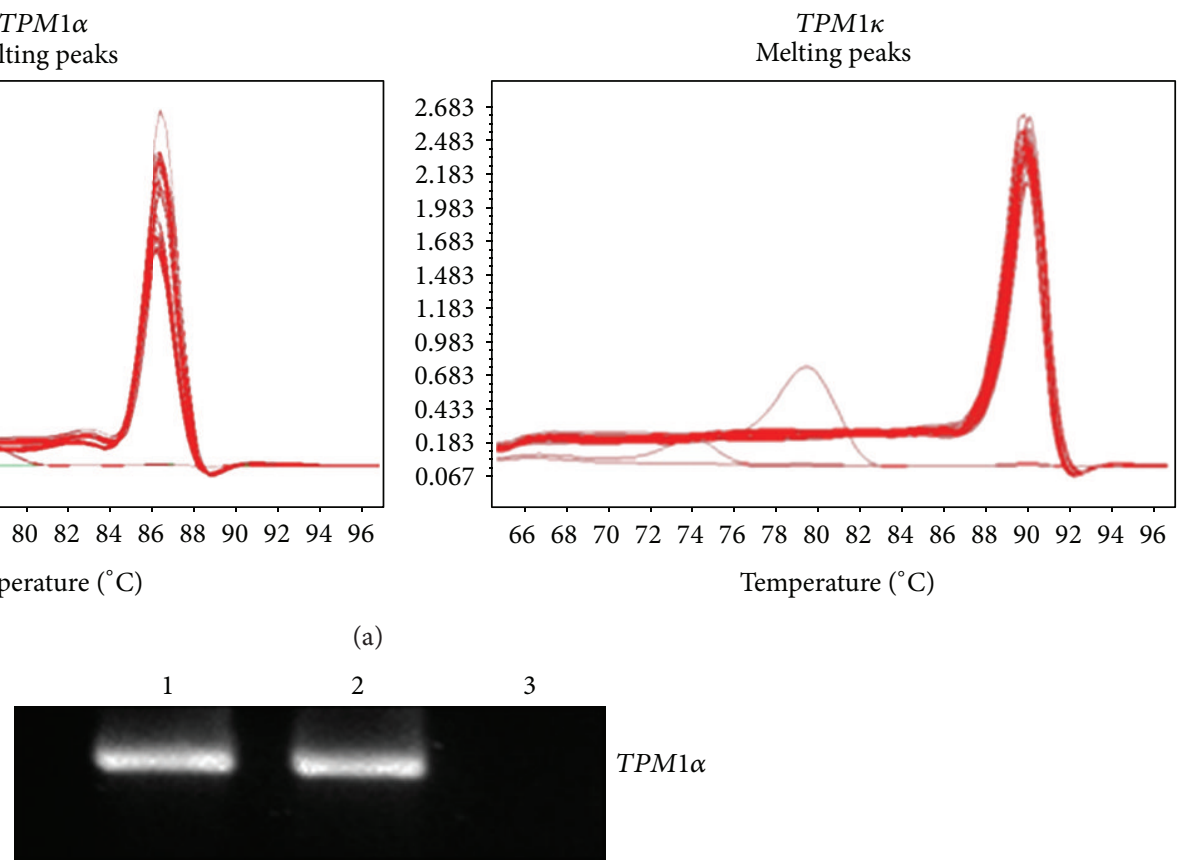

$T P M 1 \alpha$

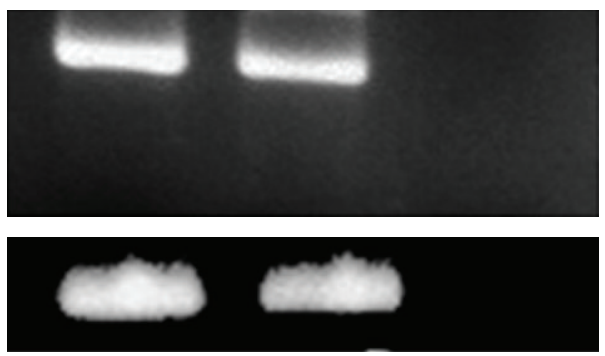

$T P M 1 \kappa$

(b)

FIGURE 4: Melt curves of SYBER green PCR and the agarose gel electrophoresis of PCR products with ethidium bromide staining. (a) Melt curves for amplified TPM $1 \alpha$ and TPM $1 \kappa$ DNA. The peaks melting at one different temperature specific for TPM $\alpha$ or TPM $\kappa$ demonstrate specific amplification of just one product for TPM1 $\alpha$ and TPM1 $\kappa$. The multiple curves represent the products from multiple replicates of the RT-PCR assay. (b) Agarose gel of PCR products with ethidium bromide staining to demonstrate amplification of a single product for TPM1 $\alpha$, TPM1 $\kappa$, and 18 S rRNA amplification. Lane 1 is heart, lane 2 is skeletal muscle, and lane 3 is for the respective primer control.

TABLE 4: qRT-PCR for mouse heart and skeletal muscle.

\begin{tabular}{lcccc}
\hline Muscle type & & $\begin{array}{c}\text { Fold differences of TPM1 } \alpha / \mathrm{TPM} 1 \kappa \\
\left(2^{\wedge}-(\mathrm{dCT})\right)^{*} \\
\text { Experiment } 2\end{array}$ & Experiment 3 & Average \\
\hline Heart & Experiment 1 & 843.08 & 879.13 & $790.75 \pm 123.1$ \\
Skeletal muscle & 650.05 & 4374.45 & 6292.72 & $5346.22 \pm 959.3$ \\
\hline
\end{tabular}

${ }^{*}$ TPM1 $\alpha$ heart: TPM1 $\kappa$ heart, $P<0.001$; TPM1 $\alpha$ skeletal muscle: TPM1 $\kappa$ skeletal muscle $P<0.001$. TPM1 $\alpha /$ TPM1 $\kappa$ heart: TPM1 $\alpha /$ TPM1 $\kappa$ skeletal muscle, $P<0.001$.

TABLE 5: Fold changes of TPM1 $\alpha$ and TPM1 $\kappa$ in heart and skeletal muscle $\left(2^{\wedge}-(\mathrm{ddCT})\right)$.

\begin{tabular}{lccccccc}
\hline & \multicolumn{2}{c}{ Ratio of TPM1 $\alpha$ in skeletal muscle $:$ heart $^{*}$} & \multicolumn{3}{c}{ Ratio of TPM1 $\kappa$ in heart : skeletal muscle ${ }^{*}$} \\
Expt 1 & Expt 2 & Expt 3 & Average \pm S.D. & Expt 1 & Expt 2 & Expt 3 & Average \pm S.D. \\
\hline 2.55 & 1.7 & 1.79 & $2.03 \pm 0.66$ & 3.38 & 5.88 & 10.04 & $6.41 \pm 4.83$ \\
\hline
\end{tabular}

${ }^{*}$ TPM1 $\alpha$ skeletal muscle : TPM1 $\alpha$ heart, $P<0.001$; TPM1 $\kappa$ heart: TPM1 skeletal muscle, $P<0.001$. 


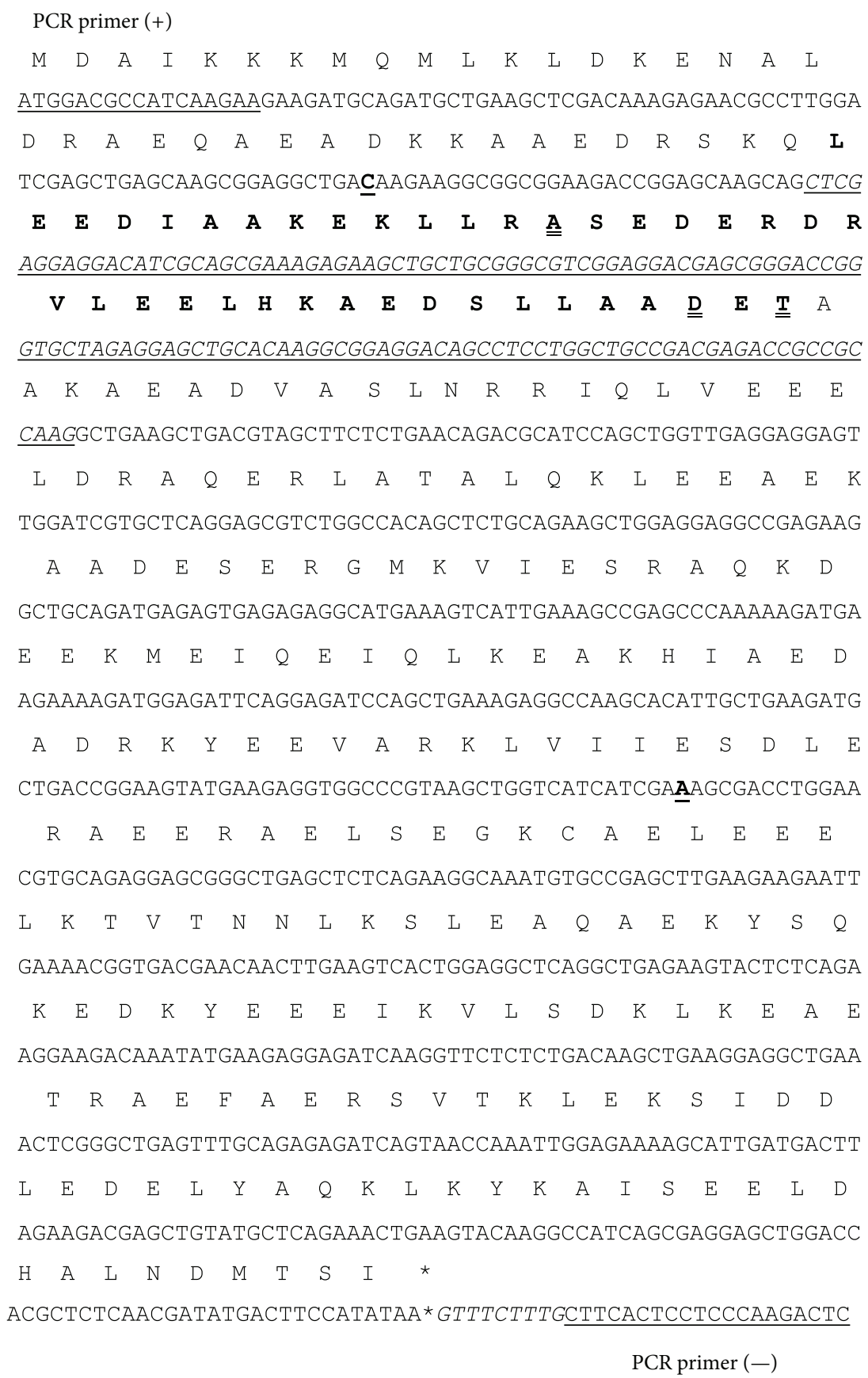

FIGURE 5: Nucleotide and deduced amino acid sequences of mouse TPM1 $\kappa$. Nucleotide sequence exon 2a is underlined and italicized. The generic primer pair that amplifies both TPM1 $\alpha$ and TPM1 $\kappa$ is underlined. Two nucleotides in TPM1 $\kappa$ in exons $1 \mathrm{a}$ and 4 were found to be different from the published sequence. TPM1 $\alpha$ sequence is made bold and underlined. These changes do not alter the amino acid sequences. Deduced protein/amino acid sequences are on the top of the nucleotide sequences. Symbol of each amino acid residue is placed on the top of the third nucleotide of each codon. Amino acid residues in exon 2a that are different form human TPMl $\kappa$ are underlined with double lines.

heart and skeletal muscle. Unfortunately, because an antimouse TPM1 $\kappa$ antibody is not available, we cannot comment on protein expression. The levels of TPM $1 \alpha$ and TPM1 $\kappa$ RNA in axolotl heart and skeletal muscle and human heart muscle are comparable. However, the level of expression of TPM1 $\kappa$ protein compared to TPM1 $\alpha$ protein is very low. This suggests a differential translational efficiency for the transcripts of the two isoforms. Hence, we cannot speculate about levels of $\mathrm{TPM} 1 \kappa$ protein in murine striated muscles.

Interestingly, the expression of $\mathrm{TPM} 1 \kappa$ protein is increased in hearts in dilated cardiomyopathy (DCM) and heart failure human patients. However, we do not know whether this increase in TPM1 $\kappa$ is the cause or consequence of the cardiac disease [12]. The immunohistochemical 
Alignment of exon 2 from human TPM1 with the homologus regions of mouse and other vertebrates

39

\begin{tabular}{|c|c|}
\hline & 1 \\
\hline usTPM1 & . D.T. \\
\hline $\mathrm{x} T P M 1 \kappa$ & 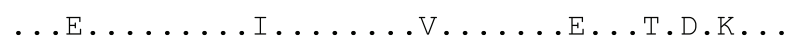 \\
\hline ChiTPM1к & ... . . QLE. \\
\hline HuTPM $1 \alpha$ & . . DELVSLQ.K.KGT . EL.KYS.A.KDAQEK.EL . KK. TD \\
\hline MusTPM1 $\alpha$ & ..DELVSLQ.K.KGT..EL.KYS.A.KDAQEK.EL . KK.TD \\
\hline $\operatorname{AxTPM} 1 \alpha$ & ..DELVALQ.K.KGT. .EL.KYS.S.KDAQEK.EL.DKK.TK \\
\hline iTP & DELVALQ . \\
\hline
\end{tabular}

FIGURE 6: Alignment of the amino acid sequences of TPM1 exon 2a or $2 \mathrm{~b}$ from various species. The top 4 sequences are $2 \mathrm{a}$ and the bottom four sequences are 2b. human ( $\mathrm{Hu}$ ), mouse (Mus), axolotl (ax), and chicken (Chi) sequences are shown. The underlined sequence in human TPM1 $\kappa$ was used to develop the antibody utilized to detect TPM1 $\kappa$ protein. The amino acid residue numbers are as shown. Conserved sequences are indicated by the $(\cdot)$.

analyses with TPM1 $\kappa$ antibody show that it is incorporated into axolotl cardiac and skeletal myofibrils and into human cardiac myofibrils [8]. Currently, the exact role $\mathrm{TPM} \kappa$ protein plays in cardiac contractility is unclear. We have shown that transfection of antisense TPM1 $\kappa$ oligonucleotides into embryonic axolotl hearts inhibits the cardiac contractility in situ and also disarrays the cardiac myofibrils [17].

Herein, for expression analysis of TPM1 $\kappa$ transcripts we performed both conventional and real-time RT-PCR with total RNA from mouse heart and skeletal muscle using isoform specific primer pairs. RT-PCR data show the expression pattern of TPM1 $\alpha$ and TPM1 $\kappa$ in mouse heart and skeletal muscle (Figure 4). We quantified the absolute copy number of TPM $\alpha$ and TPM1 $\kappa$ expressed in heart and skeletal muscle. The results presented in Tables 2 and 3 show the higher copy number of TPM1 $\kappa$ expressed in mouse heart compared to skeletal muscle. Total copy number of TPM1 $\alpha$ is higher in skeletal muscle. Hence, the difference in the ratio of copy numbers of TPM1 $\alpha$ : TPM $1 \kappa$ is even more pronounced between the two tissues. The expression of TPM1 $\alpha$ transcript is $\sim 2$ - to 3-fold higher in skeletal muscle compared to heart. On the contrary, the expression of TPM $\kappa$ is $\sim 3$-fold higher in heart compared to mouse skeletal muscle. The realtime PCR (RT-PCR) results using copy number procedure is also reflected from the qRT-PCR data using $2^{\wedge}-(\mathrm{dCT})$ method where we have used $18 \mathrm{~S}$ rRNA as the reference gene (Table 4). We conclude that expression level of TPM1 $\kappa$ transcripts is higher in mouse heart compared to skeletal muscle. Further, the expression level of TPM1 $\kappa$ compared to TPM1 $\alpha$ is significantly lower in both heart and skeletal muscle. Our analysis of qRT-PCR data by $2^{\wedge}-(\mathrm{ddCT})$ method also supports the similar conclusions (Table 5). It is worth mentioning that TPM1 $\alpha$ expressed per $\mu \mathrm{g}$ of total RNA is considerably higher in skeletal muscle relative to heart muscle in axolotl as well [8]. We do not know whether this would be a consistent finding in all vertebrates. A higher expression level of TPM $1 \alpha$ in skeletal muscle could be attributed to the higher requirement of TPM1 $\alpha$ protein in skeletal muscle or due to the higher turnover of TPM1 $\alpha$ RNA or protein in skeletal muscle. An altered dynamicity of tropomyosin in heart versus skeletal muscle may contribute to higher concentration of TPM1 $\alpha$ mRNA observed in skeletal muscle. Wang et al. [18] compared the recovery rates of mature myofibrils in avian cardiomyocytes and skeletal muscle cells transfected with avian TPM1 $\alpha$ or TPM1 $\kappa$ after photobleaching and noted the marked decreased rates of recoveries of both TPM1 isoforms in skeletal muscles compared with myofibrils in cardiomyocytes. The results suggest a lower dynamicity of tropomyosin in skeletal muscle. Hence, one can speculate that more transcripts of tropomyosin accumulate in the skeletal muscles cells or the transcriptional efficiency of the TPM1 gene is significantly higher in skeletal muscle cells. Further studies are warranted for proposing a definitive explanation.

The deduced amino acid sequences show that three amino acid residues in exon 2a (Figure 6) are different from that of human sequences. In human TPM1 $\kappa$, the 52nd amino acid residue is valine whereas it is alanine in mouse TPM1 $\kappa$. This alteration may contribute to a lower affinity of the TPM1 $\kappa$ antibody $[8,12]$ that we used for western blot analysis to detect the expression of TPM $1 \kappa$ protein in mouse heart and skeletal muscle. There is such precedence in tropomyosin literature. An antibody, ARG1, targeted toward residues 214-226 (Tyr-Ser-Gln-Lys-Glu-AspArg-Tyr-Glu-Glu-Glu-Ile-Lys) of human TPM1 $\alpha$ can recognize human TPM1 $\alpha$ but not Rat TPM1 $\alpha$. The amino acid sequence of human TPM1 $\alpha$ differs from that of rat TPM1 $\alpha$ by an Arg-to-Lys amino acid exchange in position 220 [19].

In addition, amino acid residues ${ }^{72} \mathrm{E}$ and ${ }^{74} \mathrm{~A}$ in humans are replaced by ${ }^{72} \mathrm{D}$ and ${ }^{74} \mathrm{~T}$, respectively, in mouse TPM1 $\kappa$ (Figure 6). Overexpression of human TPM1 $\kappa$ protein in a cardiac-specific manner leads to the development of DCM in transgenic mice $[12,20]$. It is to be noted that several missense mutations in exon 2b in TPM1 $\alpha$ have been implicated in human DCM [21]. Olson et al. identified two mutations in exon $2 \mathrm{~b}$ of the TPM1 gene that altered two very conserved amino acids and also reversed the charges on the surface of tropomyosin [21]. The differences of the amino acid 
residues in exon $2 \mathrm{a}$ of human TPM1 $\kappa$ and mouse TPM1 $\kappa$ (Figure 6) do not alter the net charges. At this juncture, we are unsure whether the absolute overexpression of human TPM1 $\kappa$ and/or its altered exon 2a protein relative to the mouse sequence explains the DCM-like phenotype observed in transgenic mice [12]. Obviously, one should consider creating transgenic mice with mouse TPM1 $\kappa$ to better understand the physiological relevance of TPM1 $\kappa$ protein.

\section{Conflict of Interests}

The authors declare that there is no conflict of interests regarding the publication of this paper.

\section{Acknowledgments}

The work in this laboratory was supported by Grants from American Heart Association (both National and New York State affiliate), CNY Children's Miracle Network, Syracuse, NY, Grants from Golisano Children's Hospital, Syracuse, NY, and funding from College of Health Professionals, Upstate Medical University, Syracuse, to Dipak K. Dube and the Barbara Kopp Cancer Research Fund to Bernard J. Poiesz.

\section{References}

[1] P. Gunning, G. O’Neill, and E. Hardeman, "Tropomyosinbased regulation of the actin cytoskeleton in time and space," Physiological Reviews, vol. 88, no. 1, pp. 1-35, 2008.

[2] J. P. Lees-Miller and D. M. Helfman, "The molecular basis for tropomyosin isoform diversity," BioEssays, vol. 13, no. 9, pp. 429-437, 1991.

[3] S. V. Perry, "Vertebrate tropomyosin: distribution, properties and function," Journal of Muscle Research and Cell Motility, vol. 22, no. 1, pp. 5-49, 2001.

[4] K. Pieples and D. F. Wieczorek, "Tropomyosin 3 increases striated muscle isoform diversity," Biochemistry, vol. 39, no. 28, pp. 8291-8297, 2000.

[5] M. F. Pittenger, J. A. Kazzaz, and D. M. Helfman, "Functional properties of non-muscle tropomyosin isoforms," Current Opinion in Cell Biology, vol. 6, no. 1, pp. 96-104, 1994.

[6] D. F. Wieczorek, "Regulation of alternatively spliced $\alpha$ tropomyosin gene expression by nerve extract," Journal of Biological Chemistry, vol. 263, no. 21, pp. 10456-10463, 1988.

[7] G. Schevzov, S. P. Whittaker, T. Fath, J. J. Lin, and P. W. Gunning, "Tropomyosin isoforms and reagents," Bioarchitecture, vol. 1, pp. 135-164, 2011.

[8] A. Thomas, S. Rajan, H. L. Thurston et al., "Expression of a novel tropomyosin isoform in axolotl heart and skeletal muscle," Journal of Cellular Biochemistry, vol. 110, no. 4, pp. 875-881, 2010.

[9] E. A. Luque, B. J. Spinner, S. Dube, D. K. Dube, and L. F. Lemanski, "Differential expression of a novel isoform of $\alpha$ tropomyosin in cardiac and skeletal muscle of the Mexican axolotl (Ambystoma mexicanum)," Gene, vol. 185, no. 2, pp. 175180, 1997.

[10] C. R. Denz, A. Narshi, R. W. Zajdel, and D. K. Dube, "Expression of a novel cardiac-specific tropomyosin isoform in humans," Biochemical and Biophysical Research Communications, vol. 320, no. 4, pp. 1291-1297, 2004.
[11] R. W. Zajdel, C. R. Denz, S. Lee et al., "Identification, characterization, and expression of a novel $\alpha$-tropomyosin isoform in cardiac tissues in developing chicken," Journal of Cellular Biochemistry, vol. 89, no. 3, pp. 427-439, 2003.

[12] S. Rajan, G. Jagatheesan, C. N. Karam et al., "Molecular and functional characterization of a novel cardiac-specific human tropomyosin isoform," Circulation, vol. 121, no. 3, pp. 410-418, 2010.

[13] C. R. Denz, C. Zhang, P. Jia et al., "Absence of mutation at the 50 -upstream promoter region of the TPM4 gene from cardiac mutant axolotl (Ambystoma mexicanum)," Cardiovascular Toxicology, vol. 11, no. 3, pp. 235-243, 2011.

[14] H. L. Thurston, S. Prayaga, A. Thomas et al., "Expression of Nkx2.5 in wild type, cardiac mutant, and thyroxine-induced metamorphosed hearts of the mexican axolotl," Cardiovascular Toxicology, vol. 9, no. 1, pp. 13-20, 2009.

[15] M. W. Pffaffl, "A new mathematical model for relative quantification in real-time RT-PCR," Nucleic Acids Research, vol. 29, pp. 2002-2007, 2001.

[16] K. J. Livak and T. D. Schmittgen, "Analysis of relative gene expression data using real-time quantitative PCR and the 2$\Delta \Delta$ CT method," Methods, vol. 25, no. 4, pp. 402-408, 2001.

[17] R. W. Zajdel, C. R. Denz, A. Narshi, S. Dube, and D. K. Dube, "Anti-sense-mediated inhibition of expression of the novel striated tropomyosin isoform TPM1 $\kappa$ disrupts myofibril organization in embryonic axolotl hearts," Journal of Cellular Biochemistry, vol. 95, no. 4, pp. 840-848, 2005.

[18] J. Wang, J. M. Sanger, S. Kang et al., "Ectopic expression and dynamics of TPM1 $\alpha$ and TPM1 $\kappa$ in myofibrils of avian myotubes," Cell Motility and the Cytoskeleton, vol. 64, no. 10, pp. 767-776, 2007.

[19] D. Wernicke, C. Thiel, C. M. Duja-Isac et al., " $\alpha$-tropomyosin mutations Asp175 Asn and Glu180 Gly affect cardiac function in transgenic rats in different ways," American Journal of Physiology: Regulatory Integrative and Comparative Physiology, vol. 287, no. 3, pp. R685-R695, 2004.

[20] C. N. Karam, C. M. Warren, S. Rajan, P. P. de Tombe, D. F. Wieczorek, and R. J. Solaro, "Expression of tropomyosin- $\kappa$ induces dilated cardiomyopathy and depresses cardiac myofilament tension by mechanisms involving cross-bridge dependent activation and altered tropomyosin phosphorylation," Journal of Muscle Research and Cell Motility, vol. 31, no. 5-6, pp. 315-322, 2011.

[21] T. M. Olson, N. Y. Kishimoto, F. G. Whitby, and V. V. Michels, "Mutations that alter the surface charge of alpha-tropomyosin are associated with dilated cardiomyopathy," Journal of Molecular and Cellular Cardiology, vol. 33, no. 4, pp. 723-732, 2001. 

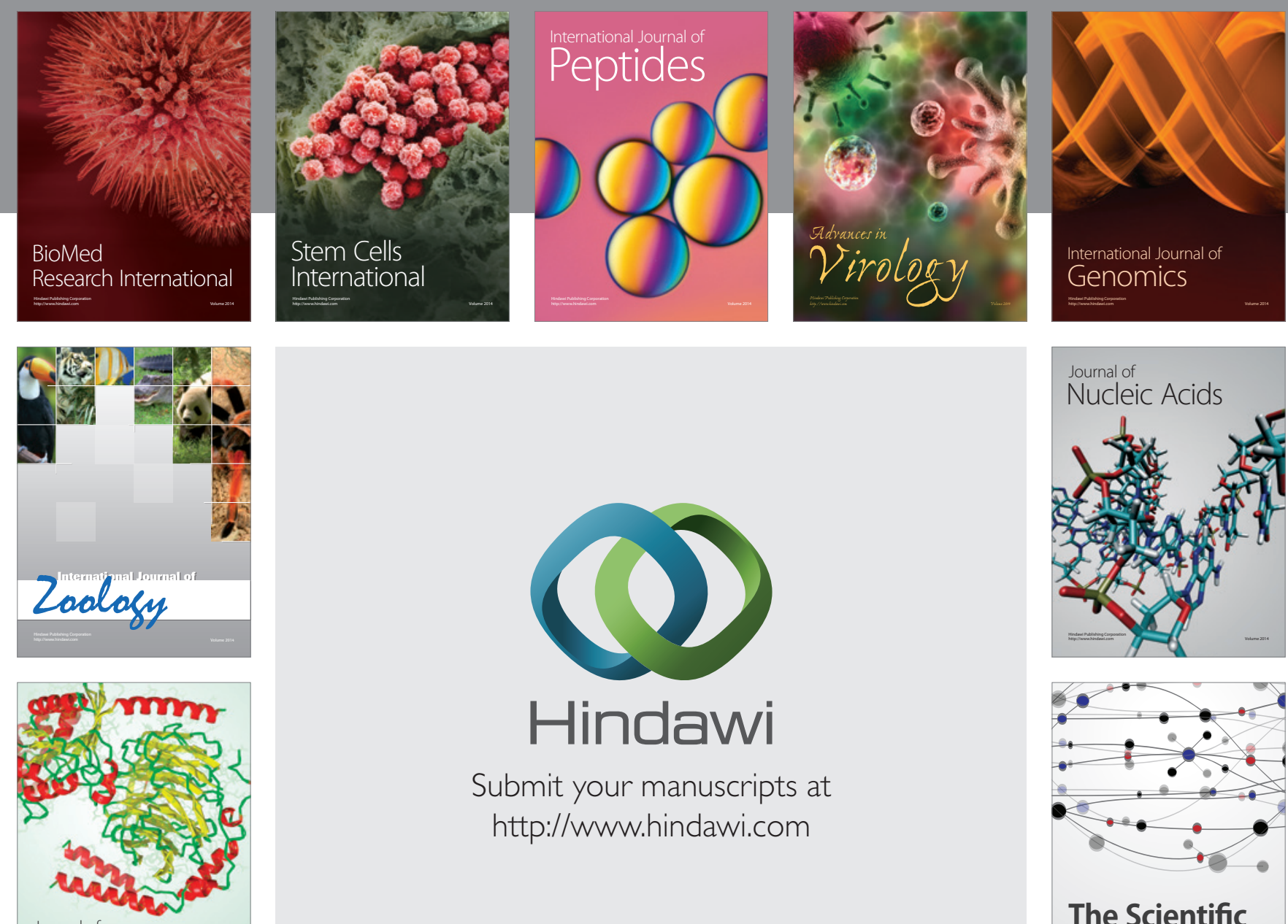

Submit your manuscripts at

http://www.hindawi.com

Journal of
Signal Transduction
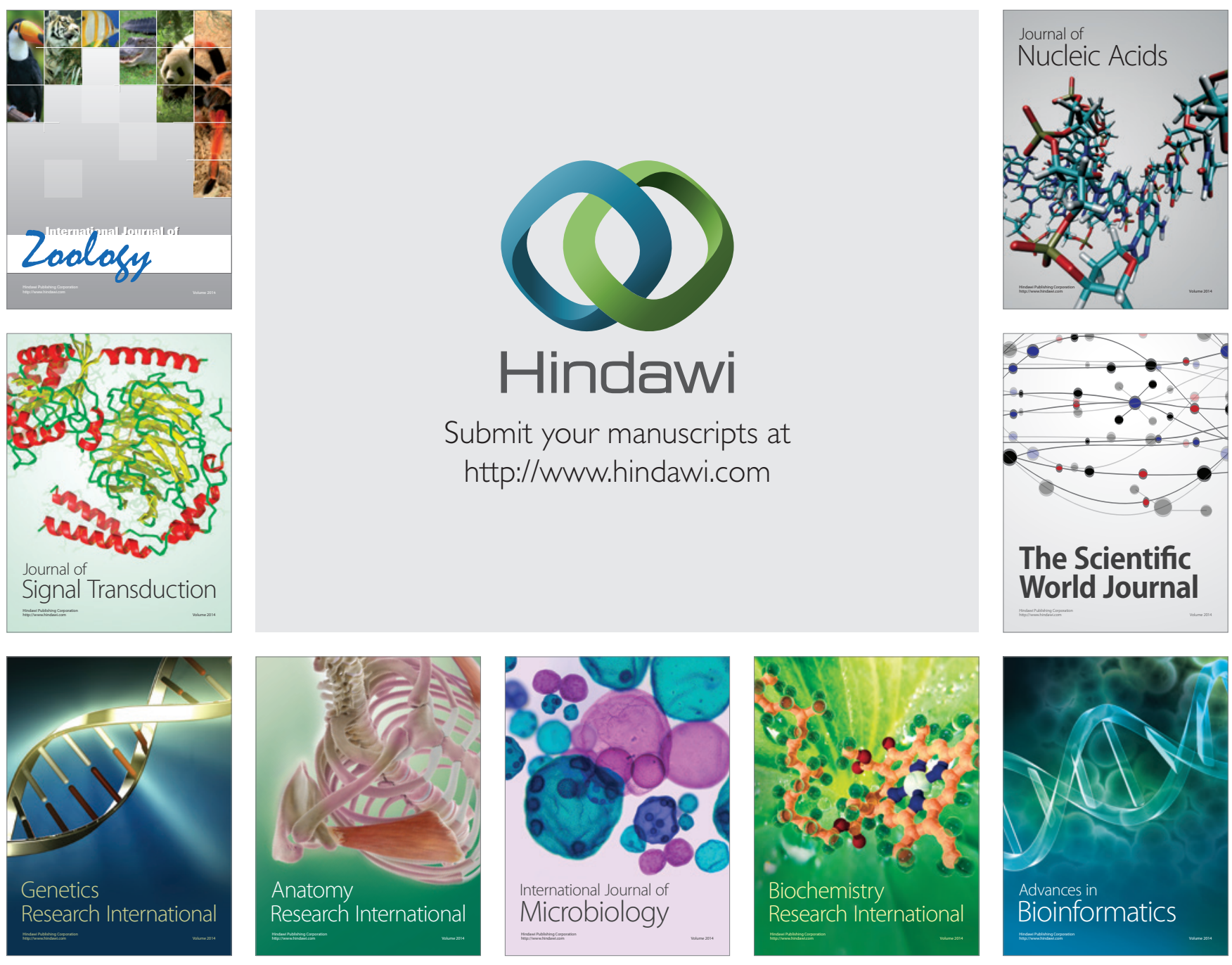

The Scientific World Journal
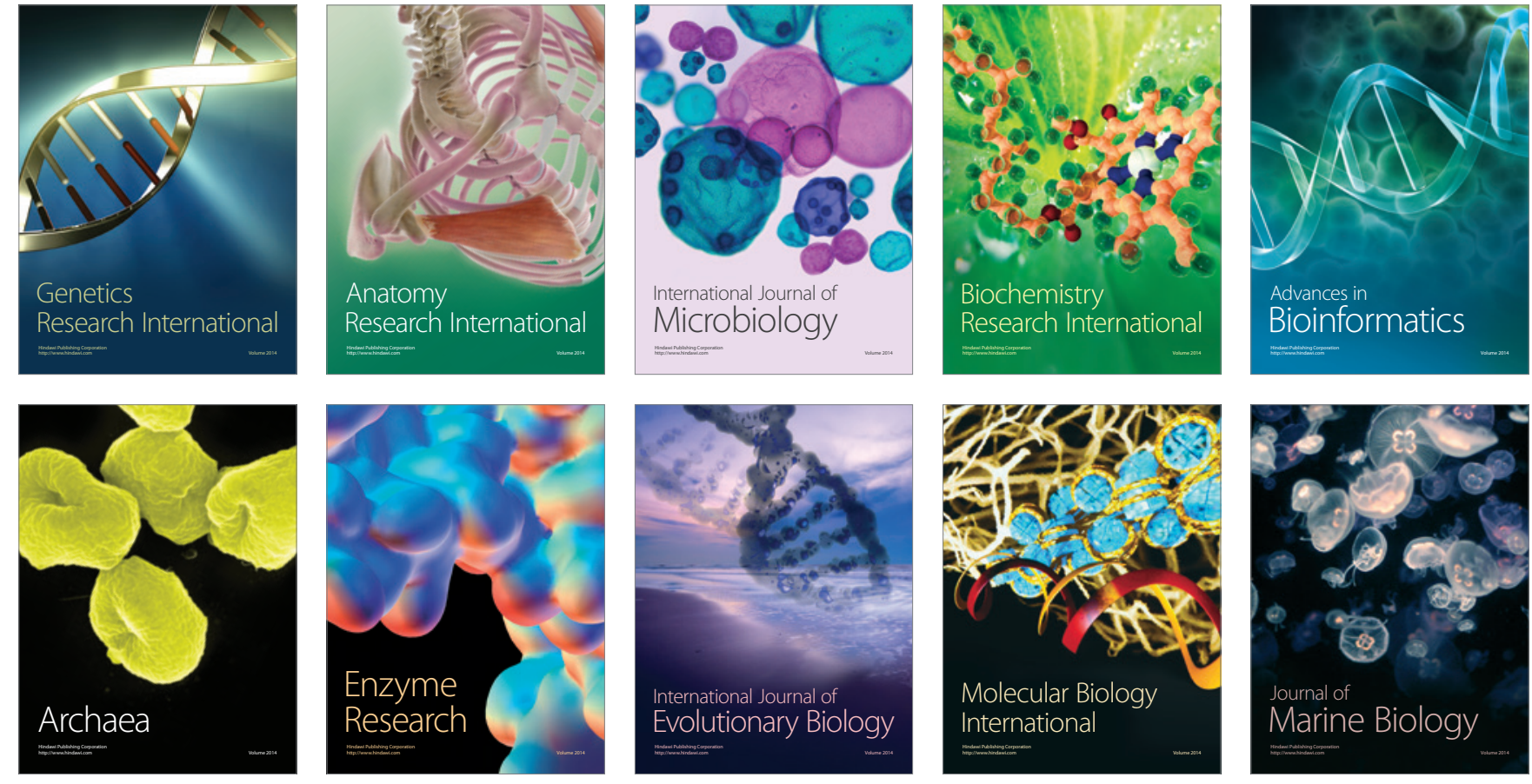